\title{
Two memorable years
}

Published at www.cmaj.ca on Dec. 4, 2009

"You will work for many years in your career - make two of them memorable."

So the advertisement for VSO (Voluntary Services Overseas) read and so, indeed, we have spent two very memorable years in Malawi. From a family practice in Kimberley, British Columbia, my husband, our two children and I moved to a small town in "The Warm Heart of Africa."

Following a very steep learning curve in tropical medicine, Chichewa language immersion and a crash course in Malawi's sometimes perplexing political health care system, I found myself in a position of consultant for a 250-bed hospital (with usually 300-400 patients), one of only two medical doctors serving a district of half a million people.

As I obviously could not do everything and my job description was delightfully vague, I was free to focus on the areas my local colleagues and I felt were most important. Routine problems were addressed by a team of medical assistants and nurses so I was consulted on only the most complicated patients.

The intensity of the medical work was unlike anything I have experienced: challenging, interesting and ever-varied, forcing me to stretch myself to the very limits of my (necessarily limited) capacities as I was called from breech deliveries to acute abdomens to meningitis to multivictim traumas. The potential to help save many lives a day was very real and often very rewarding.

But trying to manage all of these serious conditions with limited resources also meant witnessing more death and suffering than I could ever have imagined. A day could move from exhilarating to exasperating to exciting to exhausting.

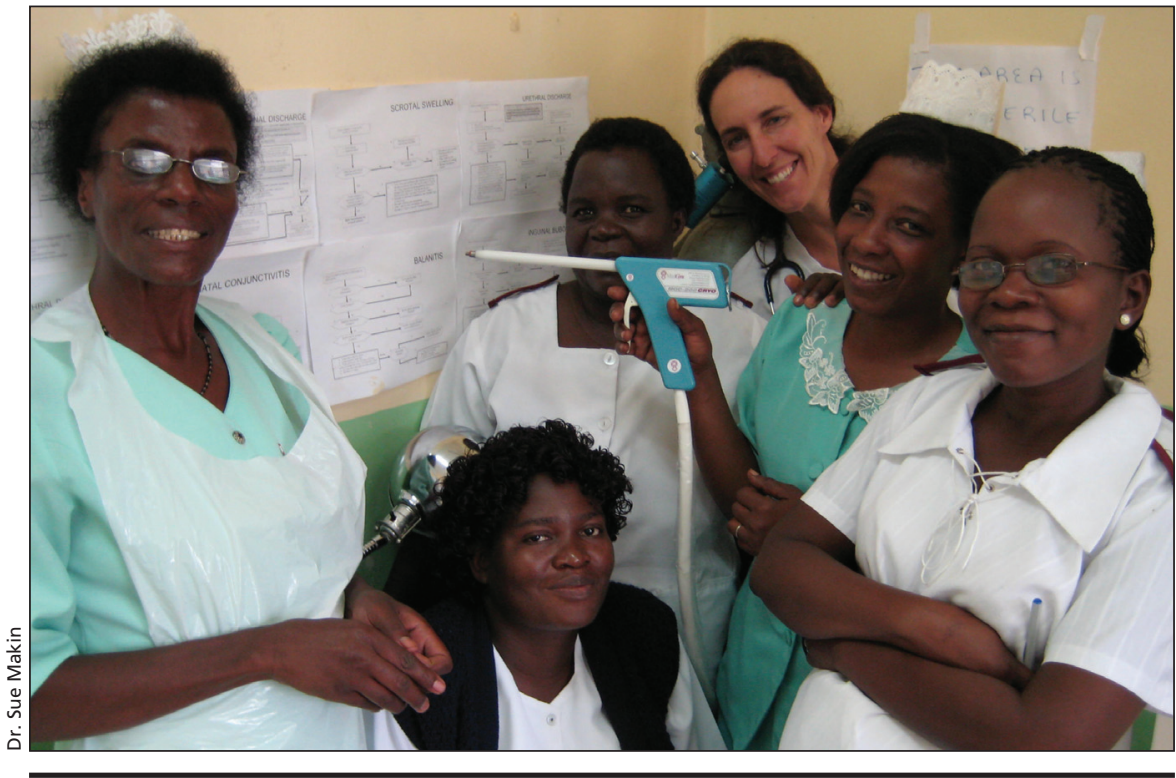

Dr. Ilona Hale (centre back) and nurses at the cervix cancer screening clinic that was established in Ntcheu District Hospital in Malawi.

The more sustainable focus of my work involved sharing skills with my local colleagues (with the sharing going both ways, of course, as I had much to learn from them) and using my different training, experience and contacts to potentially benefit patients long after I have left Malawi.

We developed new trainings, protocols, work aids, patient teaching materials and a resource library. We explored the concepts of evidenced-based medicine and patient-centred care, increased HIV-testing rates and reduced some unnecessary interventions.

One of the most substantial changes was the development of a cervix cancer screening clinic in Malawi. Cervix cancer is the leading cancer killer of women and sadly, no screening was previously available in the district. I routinely saw women with late stage cancers and had nothing to offer but palliation. Pap smears are not feasible because of the lack of infrastructure but a system called
"VIA" (visual inspection with acetic acid) is recommended as it has similar sensitivities, and lesions can be treated with cryotherapy in the same visit by nurses or clinical officers with relatively little training.

By the time I left, we had screened hundreds of women and detected a startlingly high prevalence (up to $10 \%$ in the HIV-positive population) of positive tests. Because there has been such high demand from local women and enthusiasm from the staff, I have no doubt that this clinic will continue to operate.

Many have asked and wondered why on earth we would leap off into the unknown to do something like this, leaving behind all that is comfortable and familiar.

My response now can only be: "Why wouldn't everyone?"

The inconveniences have been small, the rewards great.

Moving outside of our ordinary life and looking at it from somewhere like 
Malawi has given us an unparalleled opportunity for a different perspective and, I believe, more clarity to see what is really important. Witnessing the reality of life in a developing country has made me a more passionate advocate for global justice: there is too much that is wrong for me to sit back and ignore it any longer.

As physicians, we all have a tremendous opportunity and, some might say, a responsibility to provide care to those who need it most, even beyond our own borders. In doing so, we can find ourselves touched by the lives of those we help, sharing in their individual human dramas and so adding richness and meaning to our own lives. - Ilona Hale MD, Ntcheu District Hospital, Malawi

DOI:10.1503/cmaj.109-3125
CMAJ invites contributions to "Dispatch from the medical front," in which physicians and other health care providers offer eyewitness glimpses of medical frontiers, whether defined by location or intervention. Submissions, which must run a maximum 700 words, should be forwarded to:wayne.kondro@cmaj.ca 